\title{
AN INVESTIGATION OF THE MELTING TEMPERATURE EFFECT ON THE RATE OF SOLIDIFICATION IN POLYMER USING A MODIFIED PHASE FIELD MODEL
}

\author{
Mochamad Chalid $^{1}$, Arbi Irsyad Fikri ${ }^{1}$, Hanindito Haidar Satrio ${ }^{1}$, Muhammad Joshua Y.B. ${ }^{1}$, \\ Jaka Fajar Fatriansyah ${ }^{1, *}$ \\ ${ }^{1}$ Department of Metallurgy and Materials Engineering, Faculty of Engineering, Universitas \\ Indonesia, Kampus UI Depok, Depok 16424, Indonesia
}

(Received: June 2017 / Revised: July 2017 / Accepted: November 2017)

\begin{abstract}
A phase field model has been successfully constructed to simulate the behavior of the semicrystalline polymer solidification phenomenon. It is a model that has been widely and successfully utilized to simulate solidification phenomena in metals. However, the non-conserved phase field equation can be extended to include unique polymer parameters that do not exist in metals; for example, polymer melt viscosity and the diffusion coefficient. In order to extend this model, we incorporate free energy density and non-local free energy density based on the Harrowell-Oxtoby and Ginzburg-Landau theorems for polymer. By using the expansion principle for the higher order of binary phase field parameter, a full modified phase field equation can be obtained. The solidification phenomenon in polymer is very important to optimize the final properties of the products. Here, we use our modified equation to investigate the effect of melting temperature on the rate of solidification. It was found that the rate of solidification is correlated with melting temperature in a non-straightforward manner.
\end{abstract}

Keywords: Phase field; Polymer; Polymer product; Solidification

\section{INTRODUCTION}

The demand for geometrically complex plastic products has been rising over the past 20 years, and this trend may continue for a further 10 years. This preference for the use of plastics is partially due to their light weight (Corinaldesi et al., 2015) and the relative ease with which they can be manufactured (Atzeni et al., 2010) in comparison to other materials. In order to produce geometrically complex plastic products, it is very important to control the solidification and morphology of the melted plastic in both the injection/extrusion machine and mold. To control the solidification and morphology of melted plastic, the basic mechanism by which melted plastic becomes solid should be well understood. The basic mechanism of plastic solidification involves a connection between complicated conservation and transport laws as well as the micro phase transformation phenomena that govern the plastic/polymer melt flow and solidification/crystallization. However, it is very difficult to describe these connections analytically. Advances in computational modeling can address this difficulty and enhance the prediction of solidification and morphology during the product manufacturing process.

Solidification in polymers differs from that in metals due to the higher melt viscosity and lower thermal properties (such as melting temperature). The high melt viscosity, low melting temperature, and partial crystallization characteristics of polymers all have a major effect on

*Corresponding author's email: fajar@metal.ui.ac.id, Tel: +62-21-786 3510, Fax: +62-21-787 2350

Permalink/DOI: https://doi.org/10.14716/ijtech.v8i7.707 
solidification, and morphological evolution in the manufacturing process of polymer products. Furthermore, the complicated kinetics involved in the solidification of polymers due to their molecular chain folding adds further difficulty to the task of physically describing them, when compared to metals.

Various techniques have been used to model solidification in polymers. Lovinger et al. described single crystal growth from Poly (trifluoroethylene) in the melted phase using a diffusion mechanism explanation (Lovinger \& Cais, 1984). Micheletti and Burger (2001) examined the non-isothermal solidification of polymers using a stochastic birth-and-growth-based algorithm. Raabe and Godara (2005) examined the kinetics and topology of polymer solidification using a three-dimensional cellular automaton model. Xu et al. (2005) discussed the solidification of isotactic polystyrene single crystals using the phase field method. In this paper, intrigued by the simplicity of the phase field model, we modify a phase field model that has been established in metal counterparts and calculate the solidification rate in polystyrene.

Phase field theory has been used in diverse problems involving the microstructural evolution of materials. Phase field theory has been successfully employed to calculate the motion of interfaces and phase boundaries without explicitly tracking those interfaces (Warren et al., 2003); for example, it has been successfully used to model solidification in pure materials/compounds (Collins \& Levine, 1985) and alloys (Wheeler et al., 1992). Warren et al. developed a twodimensional phase field model of grain boundary statics and dynamics for polycrystalline materials (Warren et al., 2003). In this paper, we extend the phase field model and simulate solidification in semi-crystalline polymer under the effect of melting temperature using phase field theory.

\section{EXPERIMENTAL}

\subsection{Landau Theory}

To describe the Ginzburg-Landau (GL) theory, it is convenient to begin with the Ising model of magnetism (Provatas \& Elder, 2005). According to this model, the energy of a second microscopic system can be described in terms of a collection of magnetic spins. The system has a domain of atoms, each of which carries a magnetic spin with the value $s_{i}= \pm 1$ that shows whether an atom's magnetic moment is pointing up or down. The energy of the system can be written as

$$
E\left\{s_{i}\right\}=-\left(\boldsymbol{J} \sum_{i}^{N} \sum_{j}^{v} s_{i} s_{j}+\boldsymbol{B} \sum_{i}^{N} s_{i}\right)
$$

where $\boldsymbol{J}$ and $\boldsymbol{B}$, respectively, are the coupling constant or pure energy that adheres to each spin and its interactions, and the external magnetic field (Ruderman \& Kittel, 1954; Kittel, 2005). The first term of the equation sums up all of the energies that are caused by the interaction of each spin (" $i$ ") with all other adjacent spins ("j"), while the second term adds the energy due to the interaction of each spin with an externally imposed magnetic field $(\boldsymbol{B})$. This magnetic field in some cases can also be considered as the chemical potential of the system.

$$
\begin{aligned}
& J=\beta K, \\
& B=\beta h,
\end{aligned}
$$

and

$$
\beta=\frac{1}{k_{B} T}
$$


According to the Landau theory, the partition function of the system can be formulated from the Ising model of magnetism. The partition function of the system is given by

$$
Z=\sum_{s_{i}= \pm 1}^{N} e^{J \sum_{i}^{N} \sum_{j}^{v} s_{i} s_{j}+B \sum_{i}^{N} s_{i}}
$$

The average magnetization ("m") of the system can be considered as the order parameter $\Psi$, which is defined as (Ruderman \&Kittel, 1954)

$$
m=\Psi=\frac{1}{N} \sum_{i}^{N} s_{i}=s_{i} .
$$

Using Equation 3, we can compute the free energy function as follows

$$
\begin{gathered}
F=-k_{B} T \ln (Z), \\
F=-k_{B} T\left(J \sum_{i}^{N} \sum_{j}^{v} s_{i} s_{j}\right)-k_{B} T\left(B \sum_{i}^{N} s_{i}\right) .
\end{gathered}
$$

The free energy, $\mathrm{F}$, is called the Landau free energy. We can obtain the free energy of each spin by dividing Equation 5 by the number of spins, N (Kittel, 2005):

$$
f_{t}=\frac{F}{N}=\frac{-k_{B} T\left(J \sum_{i}^{N} \sum_{j}^{v} s_{i} s_{j}\right)}{N}+\frac{-k_{B} T\left(B \sum_{i}^{N} s_{i}\right)}{N} .
$$

From here, we can use the partition function to calculate the free energy per spin by introducing a new term:

$$
f=\frac{-k_{B} T(F)}{N} .
$$

where $F$ is the free energy function derived from the partition function. Substituting this into Equation 6 yields:

$$
f_{t}(m, T)=\frac{F(m, T)}{N}=f_{i}+f_{e}(m, T)
$$

If we consider the jump to be small, then $f_{e}(m, T)$ can be expanded using a Taylor series. However, $f_{e}(m, T)$ is an even function, thus we only need to consider the even terms.

$$
f_{e}(m, T)=f_{e}\left(m, T_{0}\right)+\frac{f_{e}^{\prime \prime} m^{2}}{2}+\frac{f_{e}^{\prime \prime \prime \prime} m^{4}}{4}+\ldots
$$

Adding this back into our equation, we obtain

$$
f_{t}(m, T)=\frac{F(m, T)}{N}=f_{i}+f_{e}\left(m, T_{0}\right)+\frac{f_{e}^{\prime \prime} m^{2}}{2}+\frac{f_{e}^{m^{\prime \prime \prime}} m^{4}}{4}+\ldots
$$

In our paper, we are interested only in the perturbation or "noise" that occurs in the system. Thus, we can omit the $f_{i}+f_{e}\left(m, T_{0}\right)$ term. One thing that needs to be considered is that first-order transitions typically occur along phases of different topological symmetry. For instance, there will be some broken symmetry. Correspondingly, a first-order transition terminates at a critical point, i.e., the point at which two co-existing phases merge into one. We can break this symmetry by adding to the equation a cubic-order term with a negative sign. 


$$
f_{t}(m, T)=\frac{F(m, T)}{N}=f_{i}+f_{e}\left(m, T_{0}\right)+\frac{f_{e}^{\prime \prime} m^{2}}{2}-\frac{f_{e}^{\prime \prime \prime} m^{3}}{3}+\frac{f_{e}^{\prime \prime \prime \prime} m^{4}}{4} .
$$

\subsection{Ginzburg-Landau Free Functional Energy}

According to the GL theory, the interaction energy between elements can be assumed to be spatially dependent, and it varies between any two elements. Let $\epsilon$ be the separation between two elements ( $i$ and $j$ ), then the mean internal energy $U$ can be defined as (Ginzburg \& Landau, 1950):

$$
U=\frac{1}{2} \sum_{i=1}^{N} \sum_{j \neq i} \grave{o}_{i j}\left(\vec{x}_{i}-\vec{x}_{j}\right) \Psi_{i}\left(1-\Psi_{j}\right)
$$

Equation 13 can be elaborated using an algebraic identity,

$$
\Psi_{i}\left(1-\Psi_{j}\right)=\left(\left[\Psi_{i}-\Psi_{j}\right]^{2}-\left[\Psi_{i}^{2}+\Psi_{j}^{2}\right]+2 \Psi_{i} s\right) / 2
$$

Assuming that $\epsilon_{i j}$ is negligible for any $\mathrm{j}>v$ and substituting Equation 3 back into equation $\mathrm{U}$, we obtain:

$$
U=\frac{1}{4} \sum_{i=1}^{N} \sum_{j \neq i} \grave{o}_{i j}\left[\Psi_{i}-\Psi_{j}\right]^{2}-\frac{1}{2} \sum_{i=1}^{N}\left(1-\Psi_{j}\right) \Psi_{i}\left(\sum_{j \neq i} \grave{o}_{i j}\right)
$$

We can add another assumption to simplify the equation, which is the interaction energy per particle $\epsilon_{i j}$ approaching $\frac{\epsilon_{i j}}{v}$. $\epsilon_{i}$ is the isotropic mean energy over.

$$
\begin{gathered}
\frac{1}{4} \sum_{j \neq i}\left[\Psi_{i}-\Psi_{j}\right]^{2}=\frac{a^{2}}{4}\left[\left(\frac{\left[\Psi_{i}-\Psi_{R}\right]^{2}}{a^{2}}+\frac{\left[\Psi_{i}-\Psi_{T}\right]^{2}}{a^{2}}\right)+\left(\frac{\left[\Psi_{i}-\Psi_{L}\right]^{2}}{a^{2}}+\frac{\left[\Psi_{i}-\Psi_{B}\right]^{2}}{a^{2}}\right)\right] \\
\frac{1}{4} \sum_{j \neq i}\left[\Psi_{i}-\Psi_{j}\right]^{2} \approx \frac{1}{2} a^{2}\left|\nabla \Psi\left(\vec{x}_{i}\right)\right|^{2} \\
F[\Psi, T]=\int_{V} \frac{1}{2}\left|W_{o} \nabla \Psi\right|^{2}+f(\Psi(\vec{x}), T(\vec{x}))
\end{gathered}
$$

\subsection{Phase Field Model}

A phase field model describes phase transformation using a crystal order parameter. The crystal order parameter distinguishes the two phases of liquid and solid in terms of $\phi . \phi$ is defined as 0 and 1 for liquid and solid respectively. The solidification process can be described as a continuous increase in the $\psi$ value from zero to one. The solidification process of polymers can be modeled based on GL theory based on the fact that GL theory incorporates the change in free energy on the boundary to explain the transformation of phase. This theory explains the solidification of polymer under the effect of decreasing temperature and distance from the nuclei. In this theory, one needs to define the anisotropy coefficient to induce solidification. In metal solidification, the anisotropy coefficient $(\varepsilon)$ will be greater than zero. However, in polymer solidification, the anisotropy coefficient $(\varepsilon)$ is zero. When the value is greater than zero, the microstructure of the material becomes dendritic, while a zero value leads to the microstructure becoming spherulitic. In this section, we derive phase field theory from the definition of free energy.

A phase field model begins with the definition of free energy. A classical phase field model of the solidification of pure material/compound has free energy that can be written as follows: 


$$
\begin{aligned}
F(\phi) & =\int f_{\text {local }}(\phi, T) d V \\
& =\int f_{\text {local }}(\phi, T)+f_{\text {grad }}(\phi, T) d V \\
& =\int f_{\text {local }}(\phi, T)+\nabla \phi d V .
\end{aligned}
$$

The free energy in the system basically consists of local free energy $f_{\text {local }}(\phi, T)$ and non-local free energy $f_{\text {grad }}(\phi, T)$. This non-local free energy $f_{\text {grad }}(\phi, T)$ can be represented using a gradient term. $\phi$ can be interpreted by phase, but here let us generally interpret $\phi$ as the crystal order parameter. The evolution of the crystal order parameter can be described as a standard GL approach:

$$
\frac{\partial \phi(r, t)}{\partial t}=\Gamma \frac{\delta F(\phi)}{\delta \phi(r, t)}
$$

where $\Gamma$ is the mobility coefficient. This coefficient is defined as inversely proportional to the melt viscosity. To accommodate the dynamics of the interfacial surface, mobility is introduced as a function of the order parameter $\Gamma(\phi)$. In the case of the dynamic of the crystal (solid)-liquid interface, the functional form of $\Gamma(\phi)$ would be more reasonable (Harrowel \& Oxtoby, 1987). They derived the one-dimensional model of the crystal-liquid interface where the time and space evolution of phase $\phi$ can be written as follows:

$$
\nabla \frac{d \phi}{d t}=-\Gamma(\phi) \nabla\left(\frac{d f}{d \phi}-K \frac{d^{2} \phi}{d x^{2}}\right)
$$

where $K$ is the kinetics. If coupling of the motions between different timescales is weak, then the interface between the two parameters $\phi_{1}$ and $\phi_{2}$ can be written as:

$$
\begin{gathered}
\frac{d^{2} \phi}{d x^{2}}+\frac{v}{\Gamma_{1}} \frac{d \phi_{1}}{d x}-\frac{d}{d \phi_{1}}\left[f_{0}\left(\phi_{1}\right)+\lambda f_{1}\left(\phi_{1}, \phi_{1}\right)\right]=0, \\
\lambda \frac{d^{2} \phi}{d x^{2}}+\frac{v}{\Gamma_{2}} \frac{d \phi_{2}}{d x}-\lambda \frac{d}{d \phi_{2}} f_{1}\left(\phi_{1}, \phi_{1}\right)=0
\end{gathered}
$$

The simplest form of $f_{1}$ and related $f_{0}$ can be written as

$$
\begin{aligned}
& f_{1}\left(\phi_{1}, \phi_{2}\right)=a_{1} \phi_{2}^{2}-a_{3} \phi_{1} \phi_{2}^{3}+\phi_{2}^{4} \\
& f_{0}\left(\phi_{1}\right)=a_{1} \phi_{1}^{2}-a_{2} \phi_{1}^{3}+\phi_{1}^{4}
\end{aligned}
$$

Equation 5.b is the functional form of free energy that is used in this paper. For polymer, Equation 5.b can be transformed to:

$$
f_{\text {local }}(\phi, T)=W\left(\frac{\zeta \zeta_{0}}{2} \phi^{2}-\frac{\zeta+\zeta_{0}}{3} \phi^{3}+\frac{\phi^{4}}{4}\right),
$$

where $W$ is the height of the energy barrier of nucleation, $\zeta$ is the unstable energy barrier, and $\zeta_{0}$ is the stable solidification potential. In our calculation, we used three polymers/plastics: polypropylene (PP), polyethylene (PE), and polystyrene (PS) with the chemical structure shown 
in Figure 1 and the physical properties shown in Table 1. According to (Xu et al., 2005), one can substitute Equations 20 and 21 into Equation 19 to obtain:

$$
\frac{\partial \psi(r, t)}{\partial t}=-\Gamma\left(W \psi(\psi-\zeta)\left(\psi-\zeta_{0}\right)-\kappa_{2} \nabla_{2} \psi\right)
$$

Assuming the process occurred in one dimension and was observed from a moving frame reference with a uniform velocity of $v=\partial \psi / \partial t$, Equation 25 can be re-written as:

$$
\kappa^{2} \frac{d^{2} \psi}{d x^{2}}+\frac{v d \psi}{\Gamma d x}+\frac{\partial f}{\partial \psi}=0 .
$$

According to (Harrowel \& Oxtoby, 1987), by setting the boundary condition of $\psi \rightarrow \zeta_{0}$ as $x \rightarrow$ $-\infty$ and $\psi \rightarrow 0$ as $x \rightarrow+\infty)$, we obtained

$$
\psi(\mathrm{z})=\frac{\zeta_{0}}{1+\exp \left(z \zeta_{0} \sqrt{\frac{W}{2 \kappa^{2}}}\right.}
$$
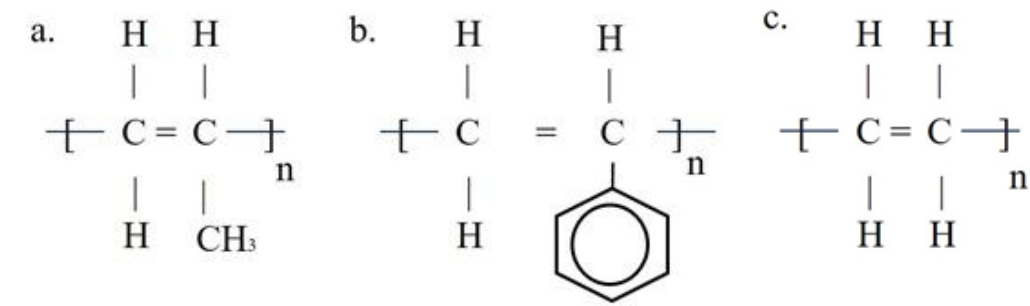

Figure 1 The chemical structure of: (a) polypropylene; (b) polystyrene; and (c) polyethylene

Table 1 The thermophysical parameters of polymers (melting temperature and crystallization temperature)

\begin{tabular}{cccc}
\hline Polymer & $\operatorname{Tm}_{0}\left({ }^{0} \mathrm{C}\right)$ & $\operatorname{Tm}\left({ }^{0} \mathrm{C}\right)$ & $T_{c}\left({ }^{0} \mathrm{C}\right)$ \\
\hline PS & 243 & 229 & 195 \\
PE & 141 & 133.4 & 120 \\
PP & 167 & 158 & 123 \\
\hline
\end{tabular}

\section{METHODOLOGY}

For each polymer, we input a set of different $v$ values into Equation 27 and used the change of solidification surface at time $t$ to plot Figure 2, which could be plotted using a standard home PC. In this calculation, it is assumed that the mobility value of all polymers is $10^{5}$ because at this stage we are interested only in the thermal gap between the melting temperature and the crystallization temperature. All of the thermophysical parameters can be found in a polymer database. The thermodynamic parameters such as kinetic coefficient, density, free energy, etc are taken from $\mathrm{Xu}$ et al. (2005).

\section{RESULTS AND DISCUSSION}

Let us discuss the results obtained from Equation 27. Figure 2 shows the change of solidification surface in respect of time. The dots are obtained through calculations and the lines are fitted by assuming that the growth of crystal is linear at a small value of $t$. Our calculation shows that PP has the highest solidification rate (from the gradient), followed by PS and PE. This can be 
explained as follows. The solidification rate is dependent on the ratio between the difference of melting temperature-crystallization temperature and equilibrium melting temperature ( $\left.\left(T_{m}-T\right) / T_{m}^{0}\right)$. This parameter is related to the heat dissipation that connects to the growth of crystal. PP has a higher value for this parameter compared to the other two polymers. This shows that in PP, heat dissipates faster and thus yields a high solidification rate. It can thus be seen that $\mathrm{PP}$ has a high relative difference between its melting temperature and crystallization temperature. Thus, to achieve equilibrium, PP needs to both dissipate heat faster and crystallize faster.

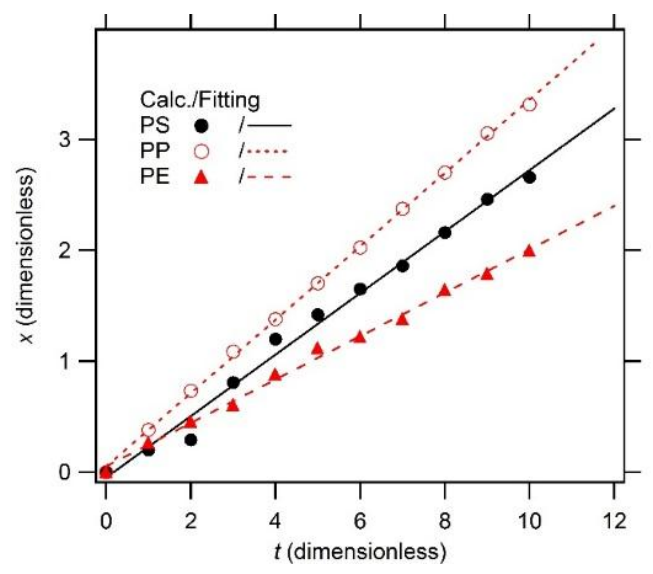

Figure 2 The change of solidification surface, $x$, in respect of time, $t$

The relation between the solidification rate and melting temperature is shown in Figure 3 . Generally, a higher melting temperature yields a higher solidification rate, except for PS. However, the ratio between the difference of melting temperature - crystallization temperature and equilibrium melting temperature $\left(\left(T_{m}-T\right) / T_{m}^{0}\right)$ is the one that relates directly to the solidification rate as this variable reflects the heat dissipation rate for each polymer. For this ratio, $\mathrm{PP}$ has a much higher value than the other two polymers and thus yields a high solidification rate despite its low melting temperature. In this calculation, we did not consider melt viscosity/mobility coefficient, which we suspect may change the results. However, it is difficult to predict the mobility coefficient as it may only be accessible via an NMR experiment. By assuming that the value of mobility is similar for every polymer, it is reasonable to use our approach.

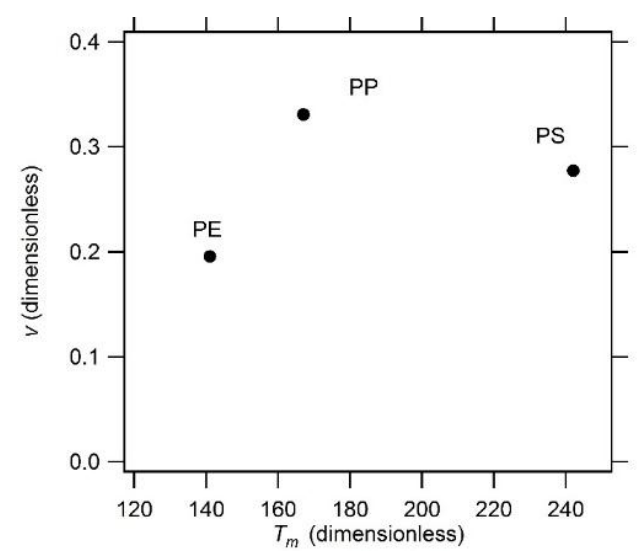

Figure 3 The difference in velocity of solidification, $v$, in respect of melting temperature, $T_{m}$ 


\section{CONCLUSION}

We calculated that PP has the highest solidification rate (from the gradient), followed by PS and $\mathrm{PE}$. This is due to the relatively large difference between the melting temperature and crystallization temperature for PP. Thus, to achieve equilibrium, PP needs to dissipate heat faster and crystallize faster. Generally, a higher melting temperature yields a higher solidification rate, except for PS. This shows that the melting temperature is not directly related to the solidification rate. There is a direct relation, however, for the ratio between the difference of melting temperature-crystallization temperature and equilibrium melting temperature $\left(T_{m}-T\right) / T_{m}^{0}$.

\section{ACKNOWLEDGEMENT}

We are grateful to and thank DRPM UI for the PITTA 2017 research grant that made it possible to conduct this research.

\section{REFERENCES}

Atzeni, E., Iuliano, L., Minetola, P., Salmi, A., 2010. Redesign and Cost Estimation of Rapid Manufactured Plastic Parts. Rapid Prototyping Journal, Volume 16(5), pp. 308-317

Collins, J.B., Levine, H., 1985. Diffuse Interface Model of Diffusion-limited Crystal Growth. Physical Review B, Volume 31(9), pp. 6119-6122

Corinaldesi, V., Donnini, J., Nardinocchi, A., 2015. Lightweight Plasters Containing Plastic Waste for Sustainable and Energy-efficient Building. Construction and Building Materials, Volume 94, pp. 337-345

Ginzburg, V.L., Landau, L.D., 1950. The Theory of Superconductivity. Zh. Eksp. Teor. Fiz, Volume 20, pp. 1064-1082

Harrowell, P.R., Oxtoby, D.W., 1987. On the Interaction between Order and a Moving Interface: Dynamical Disordering and Anisotropic Growth Rates. Journal of Chemical Physics, Volume 86(5), pp. 2932-2942

Kittel, C., 2005. Introduction to Solid State Physics. Wiley

Lovinger, A.J., Cais, R.E., 1984. Structure and Morphology of Poly(trifluoroethylene). Macromolecules, Volume 17(10), pp. 1939-1945

Micheletti, A., Burger, M., 2001. Stochastic and Deterministic Simulation of Non-isothermal Crystallization of Polymers. Journal of Mathematical Chemistry, Volume 30(2), pp. 169193

Provatas, N., Elder, K., 2005. Ising Model of Magnetism. In: Phase-Field Methods in Material Science and Engineering, Wiley-VCH, New York, pp. 11-14

Raabe, D., Godara, A., 2005. Mesoscale Simulation of the Kinetics and Topology of Spherulite Growth during Crystallization of Isotactic Polypropylene (iPP). Modelling and Simulation in Materials Engineering, Volume 13, pp. 733-751

Ruderman, M.A., Kittel, C., 1954. Indirect Exchange Coupling of Nuclear Magnetic Moments by Conduction Electrons. Physical Review, Volume 96(1), pp. 99-102

Warren, J.A., Kobayashi, R., Lobkovsky, A.E., Carter, W.C., 2003. Extending Phase Field Models of Solidification to Polycrystalline Materials. Acta Materialia, Volume 51(20), pp. 6035-6058

Wheeler, A.A., Boettinger, W.J., McFadden, G.B., 1992. Phase-field Model for Isothermal Phase Transition in Binary Alloys. Physical Review A, Volume 45(10), pp. 7424-7439

$\mathrm{Xu}, \mathrm{H}$., Matkar, R., Kyu, T., 2005. Phase-field Modelling on Morphological Landscape of Isotactic Polystyrene Single Crystal. Physical Review E, Volume 72(1), 011804 